\title{
La Structuration des Systèmes Associatifs Locaux de Bien-Être
}

\author{
Yvan Comeau*
}

\begin{abstract}
$\mathrm{R}$ locais baseando-se em dados empíricos da província do Quebec, no Canadá. A informação, de natureza quantitativa e qualitativa, revela uma grande diversidade dos sistemas associativos locais. Assim, num segundo momento, o texto apresenta uma análise das dimensões que contribuem para essa diversidade. Dois tipos de fenómenos estruturam essa diversidade das associações: os fenómenos estruturais e os fenómenos interpretativos. De entre os fenómenos estruturais, salienta-se o território, a idade das associações, a sua dimensão e o tipo de uso dos serviços. No que diz respeito aos fenómenos interpretativos, encontramos a dinâmica das relações sociais e dos esquemas de interpretação construídos. Finalmente, o texto sublinha o contexto e a capacidade de inovação social destas associações.
\end{abstract}

Palavras-chave: associações; participaçâo; democracia; economia social; movimentos sociais; políticas sociais; estado-providência.

\section{Introduction}

Depuis le début de l'industrialisation (Mayer, 2002), il existe dans les quartiers urbains et les communautés rurales, des systèmes associatifs de bien-être, en interface avec les services publics et privés, et plus ou moins liés à d'autres systèmes associatifs locaux, contribuant ainsi à former des regroupements territoriaux et sectoriels plus larges. Ces systèmes mènent des activités de natures sociale, sanitaire, éducative et préventive, et contribuent à la production de richesse monétaire et non monétaire ${ }^{1}$ (Viveret, 2001). Leurs activités comportent des volets relationnel (accueil, écoute, relation d'aide, accompagnement et suivi), d'aide (entraide et dépannages alimentaire et vestimentaire), d'hébergement, de répit, de garde et d'éducation; lorsque des problématiques sont clairement identifiées par les organismes, celles-ci concernent des problèmes économiques (pauvreté et chômage), de santé (santé physique et mentale) et d'insertion sociale (personnes handicapées et analphabètes, notamment).

Les associations elles-mêmes ne s'attribuent pas toutes une mission de bien-être. Il s'avère tout de même pertinent, dans une perspective de compréhension générale des politiques sociales, de mettre l'emphase sur cette mission. En ce sens, le but de cet article consiste d'abord à démontrer en quoi ces associations orientées vers le bien-être forment «système», au sens d'un ensemble régularisé de pratiques et de relations sociales (Giddens, 1997). Puis, l'article identifie un certain nombre de phénomènes façonnant ces systèmes selon diverses configurations: des systèmes associatifs locaux de bien-être démontrent davantage d'ouverture que d'autres à l'inclusion d'une variété d'acteurs; des systèmes réussissent à agréger des ressources plus considérables que d'autres; d'autres systèmes accordent des conditions de travail plus intéressantes que

\footnotetext{
Professeur École de Service Social, Université Laval. Cøntacto: yvan.comeau@svs.ulaval.ca

' Dans la région de Chaudière-Appalaches (350 000 résidents), le système associatif local de bien-être procure 3127 emplois en $2000-2001$ (32 $440221 €$ en salaires) et réalise des dépenses annuelles de $72776495 €$. Dans la mesure $\bullet$ il serait possible de traduire en monnaie toute la richesse produite, le système procure un encadrement gratuit de personnes pour un montant de $5774519 €$ et mobilise des bénévoles qui fournissent un travail gratuit équivalant à $4482248 €$ par année. La richesse produite doit également tenir c•mpter du temps des 12912 personnes participant aux assemblées générales et des 4101 personnes présentes aux conseils d'administration, sans compter les réseaux d'établissements, d'entreprises et de personnes qui se sont constitués autour de ce système (Comeau, 2003).
} 
d'autres. C'est à partir de recherches empiriques à caractère territorial et $d$ 'analyses statistiques que sont faits les constats et le repérage des phénomènes structurants ${ }^{2}$.

De manière un peu plus précise, on peut définir un système associatif local de bien-être comme étant l'ensemble des associations offrant des services spécifiques en vue d'améliorer la santé et la qualité de vie des résidents d'un territoire au moyen d'activités variées et dans une perspective d'intérêt général. La diversité caractérise un système associatif local particulier et les systèmes entre eux. Cette variété s'explique d'abord par l'espace puisqu'un territoire donné offre des opportunités et des contraintes contribuant à favoriser telle ou telle caractéristique des systèmes associatifs locaux. Cette diversité se développe également dans la durée parce que dans une société située et datée, la question sociale se pose d'une certaine manière et parce que les politiques sociales y apportent des réponses variées et changeantes (Esping-Andersen, 1996 et 1999; Vaillancourt, 2002). En outre, leur diversité tient à la variété des acteurs qu'on y retrouve, aux divers cadres d'interprétation que ceux-ci proposent concernant les problèmes de bien-être et leur solution, et à la dynamique sociale qu'ils construisent (Benford et Hunt, 2001).

\section{Les Caractéristiques Institutionnelles des Systèmes Associatifs Locaux}

De manière générale, les systèmes associatifs locaux peuvent être décrits à partir des dimensions institutionnelle et organisationnelle ${ }^{3}$. La composante institutionnelle privilégie les rapports entre les acteurs, afin de reconnaître les pressions politiques qui agissent sur et dans les organismes. Il existe donc non seulement une composante institutionnelle interne aux organismes, mais aussi une composante externe. Les phénomènes institutionnels internes sont reliés aux règlements des organismes, à la mission, au réseau et à la prise de décisions; c'est le système politique qu'on retrouve dans les règles du jeu et les coutumes particulières. Les phénomènes institutionnels externes, quant à eux, sont reliés à la notion d'espace public dans lequel se manifestent l'État, les forces du marché et la société civile (Laville et Sainsaulieu, 1997). La question des rapports entre les systèmes associatifs locaux de bien-être et l'État se trouve donc posée. L'État réglemente, finance et dispense en partie les services de bien-être; les systèmes associatifs locaux offrent une partie des services, avec une faible possibilité de générer des revenus de manière autonome mais avec une grande capacité de repérer les besoins des populations et d'y répondre de manière conviviale. Dépendamment des secteurs, des modalités de rencontre entre l'État et les systèmes associatifs, et de la capacité d'agir (empowerment) des associations, les rapports entre eux vont du conflit ouvert à la collaboration franche, en passant par tout l'éventail des rapports partenariaux ${ }^{4}$, incluant l'instrumentalisation de certaines associations.

Sur le plan institutionnel, on observe, en premier lieu, dans les systèmes associatifs locaux de bien-être, la présence de statuts juridiques

\footnotetext{
${ }^{2}$ Ces enquêtes touchent les territoires de Montréal (153 organismes investigués en 1995 par Mathieu et al., 2001), de l'Estrie (4 organismes d'aide alimentaire analysés en profondeur en 2000 par Mercier et Métivier, 2003), de Chicoutimi (36 organismes étudiés en 1997 par Tremblay et Tremblay, 1998), du Bas Saint-Laurent (241 organismes de la nouvelle économie sociale considérés en 1998 par Saucier et Thivierge, 1999) et de Chaudière-Appalaches (250 organismes répondent, en 2001, au questionnaire de Comeau, 2003). Ces travaux ne font pas référence à la notion de système associatif local de bien-être, mais ils partagent la préoccupation de recueillir des informations auprès d'un nombre important d'associations et prétendent, pour la plupart, à une certaine représentativité de l'ensemble de ces associations sur un territoire.

${ }^{3}$ La dynamique des rapports sociaux se reporte à deux leviers du changement: les ententes et les règles en vigueur, d'une part, et l'acquisition de ressources, d'autre part. En ce sens, Alain Touraine (1993: 58-67) distingue deux dimensions fondamentales de l'analyse sociologique: institutionnelle et organisationnelle. La dimension institutionnelle se réfère au «système politique» qui permet de transformer l'action historique et les conflits «en un corps de décisions et de lois» et en «des mécanismes de formation des décisions légitimes» (Touraine, 1993: 59). La dimension organisationnelle englobe le «système des moyens» et les «techniques» (Touraine, 1993: 62), autrement dit les modalités de production de biens et de services, et les ressources.

${ }^{4}$ Ces rapports sont extrêmement variés et dépendent notamment de la nature des établissements en cause: les établissements de services sociaux réfèrent des personnes dans les systèmes associatifs locaux et peuvent, à l'occasion, leur fournir un support technique et diverses formes d'appui; les hôpitaux peuvent également leur recommander des personnes; les établissement scolaires y placent des stagiaires; des municipalités contribuent à leur financement et procèdent à des échanges de services; etc. (Tremblay et Tremblay, 1998: 114). Dans le domaine de l'aide alimentaire, les liens de collaboration entres les établissements publics et les systèmes associatifs locaux sont diversifiés et nombreux; les premiers contribuent à la prestation de services, à l'octroi de main-d'œuvre, au financement et sont présents dans le système politique interne des seconds (Mercier et Métivier, 2003: 73-74).
} 
correspondant plus souvent à celui de compagnie à but non lucratif qu'à celui de coopérative ${ }^{5}$. Cette distinction permet d'introduire deux composantes majeures d'un tel système: les organismes communautaires (communément désigné «le communautaire») et les entreprises de la nouvelle économie sociale ${ }^{6}$. Ces deux entités comportent bien sûr des différences entre elles. Ainsi, sur le plan juridique, les coopératives créées par les «nouveaux» mouvements sociaux des années 1970 appartiennent à la nouvelle économie sociale, tandis que les corporations à but non lucratif sont associées à la fois au communautaire et à la nouvelle économie sociale. Sur le plan économique, cette dernière tire du marché une partie importante de ses revenus, ce qui est moins souvent le cas du «communautaire» qui rejoint des populations éprouvant des problèmes sociaux particuliers et dont les services comportent une «gratuité», comme le sont d'une certaine manière les services publics de santé et de services sociaux. Les ressemblances suivantes demeurent tout de même suffisamment nombreuses pour qu'on puisse les considérer toutes deux en tant que composantes des systèmes associatifs locaux de bien-être.

En deuxième lieu, les systèmes associatifs locaux de bien-être se caractérisent, sur le plan institutionnel, par un fonctionnement démocratique misant sur une assemblée générale et un conseil d'administration. La très grande majorité des organismes tiennent une assemblée générale annuelle; la presque totalité des organismes gèrent les choses courantes avec un conseil d'administration ${ }^{7}$. Mis à part les conseils exécutifs qui sont beaucoup plus rares que les comités de travail ${ }^{8}$, il n'y a pas pratiquement pas d'autres instances d'expression et de décision; un lieu permettant aux usagers de s'exprimer est l'instance la plus courante ${ }^{9}$.

En troisième lieu, on remarque une implication importante des femmes dans la structure politique des systèmes associatifs locaux. Globalement, ce sont majoritairement des femmes que l'on retrouve dans les conseils d'administration ${ }^{10}$. Toutefois cette situation change lorsque l'on considère les secteurs d'activités, en particulier dans la nouvelle économie sociale, où il existe des secteurs dont les conseils d'administration sont à dominante féminine et d'autres à dominante masculine ${ }^{11}$.

En quatrième lieu, les instances décisionnelles des systèmes associatifs locaux de bien-être font une place habituellement plus importante aux personnes qui utilisent les services plutôt qu'à celles qui les produisent ${ }^{12}$, quoique la situation

\footnotetext{
${ }^{5}$ Les données révèlent que les organismes optent très majoritairement pour le statut de compagnie à but non lucratif: 99,3\% des organismes communautaires au Québec contactés par Bourdon et al. (2001); 97,2\% des organismes investigués dans Chicoutimi (Tremblay et Tremblay, 1998: 23); 87,2\% des organismes de Chaudière-Appalaches (Comeau, 2003: 49); 62,9\% de l'économie sociale du Bas-Saint-Laurent (Saucier et Thivierge, 1999: 22).

${ }^{6}$ La distinction entre le communautaire et la nouvelle économie sociale repose ici sur le statut juridique et la nature des activités. Ce critère amène à décider qu'un organisme appartient au communautaire lorsqu'il n'est pas une coopérative et qu'il consacre moins de $50 \%$ de ses dépenses et de son temps à certaines activités comportant la possibilité d'obtenir des revenus à partir de la vente de services et de biens: garde de la petite enfance, loisir social, service d'aide à domicile, immobilier locatif, ressourcerie (récupération et réutilisation), entreprise culturelle ou artistique, média communautaire, transport collectif, entreprise d'insertion, centre de travail adapté, joujouthèque, restauration, centre de ressources périnatales, gestion faunique et entreprise collective industrielle (Comeau, 2003).

7 À cet effet, les données précises sont les suivantes. À Chicoutimi, une assemblée générale fonctionne dans $83 \%$ des organismes, alors que le conseil d'administration y œuvre dans 91\% des cas (Tremblay et Tremblay, 1998: 39). Dans Chaudière-Appalaches, 88\% des organismes tiennent une assemblée générale et 96,4\%, d'entre eux ont un conseil d'administration (Comeau, 2003: 59).

${ }^{8}$ Ainsi, on retrouve un conseil exécutif (instance émanant du conseil d'administration afin de donner suite aux affaires courantes) dans moins de la moitié des entreprises d'aide domestique, dans moins de $14 \%$ des Centres de la petite enfance («crèches» fonctionnant, pour la majorité, selon un mode associatif) et dans le quart seulement d'une catégorie d'organismes regroupant les Centres de travail adapté et les entreprises d'économie sociale en aménagement forestier (Comeau et al., 2001: 71).

${ }^{9}$ C'est effectivement le cas pour 15,6\% des organismes dans Chaudière-Appalaches (Comeau, 2003: 68).

10 À Chicoutimi, 68,1\% des membres de conseil d'administration sont des femmes (Tremblay et Tremblay, 1998: 32). Dans Chaudière-Appalaches, un conseil d'administration moyen compte 7,7 personnes (4,5 femmes et 3,2 hommes) (Comeau, 2003: 66).

${ }^{11}$ Deux fois plus de femmes que d'hommes sont présentes dans les conseils d'administration des entreprises d'aide domestique et quatre fois plus de femmes siègent aux conseils d'administration des Centres de la petite enfance. Le phénomène est complètement inversé pour les conseils d'administration d'organismes regroupés dans la catégorie des Centres de travail adapté et les entreprises d'économie sociale en aménagement forestier. Le nombre moyen de sièges occupés par des hommes y est quatre fois supérieur à ceux occupés par des femmes. Quant aux organismes de la catégorie «autres» (coopératives funéraires, entreprises d'insertion, ressourceries, entre autres), on y retrouve également davantage d'hommes dans les conseils d'administration (Comeau et al., 2001: 70).

12 Moins du tiers des organismes de Chaudière-Appalaches permettent aux usagers, aux employés (à peine 18\% des organismes) et à des partenaires (les syndicats notamment) d'être présents à cette instance (Comeau, 2003: 63).
} 
varie d'un secteur à l'autre ${ }^{13}$. En ce qui concerne les acteurs du milieu, on constate que leur implication dans les instances décisionnelles de la nouvelle économie sociale est bien réelle ${ }^{14}$.

En sixième lieu, les systèmes associatifs locaux de bien-être offrent des conditions de travail relativement peu avantageuses et ce, en termes absolus ${ }^{15}$ et relatifs lorsqu'on les compare à celles des secteurs public et privé, bien qu'il puisse parfois être plus avantageux de travailler dans un système associatif local de bien-être que dans le secteur privé du commerce en détails tout particulièrement ${ }^{16}$. À l'instar du marché du travail habituel, le salaire horaire moyen varie selon la fonction exercée, la scolarisation et le type d'organismes ${ }^{17}$. Aucune recherche n'a réussi à établir des différences salariales entre les sexes pour les mêmes fonctions et dans les mêmes secteurs ${ }^{18}$. Pour la représentation des employés, les syndicats sont peu présents dans le système, bien que la situation varie lorsque l'on considère les secteurs d'activités ${ }^{19}$.

\section{Les Caractéristiques Organisationnelles des Systèmes Associatifs Locaux}

Bien qu'elle possède une autonomie relative, la dimension organisationnelle est influencée par les institutions envisagées comme ensemble de normes et de règles qui cristallisent l'issue des conflits que se livrent les acteurs en périphérie et à l'intérieur des systèmes associatifs locaux de bien-être pour l'obtention de ressources, entre autres choses. En ce sens, la composante organisationnelle est en partie déterminée par des phénomènes institutionnels. Concrètement, la composante organisationnelle concerne les moyens mis en œuvre par l'organisme pour atteindre les objectifs. Il s'agit principalement de phénomènes internes qui touchent les ressources financières et humaines, la coordination des activités, la production, et la consommation des services et des biens. La dimension organisationnelle s'avère pertinente pour considérer l'autonomie et l'implication des producteurs et des usagers dans

13 Ainsi, dans le communautaire, les organismes des secteurs «santé et services sociaux» et «éducation» permettent plus souvent aux employés de siéger au conseil d'administration que les organismes des autres secteurs (Bourdon et al., 2001: 18). Dans la nouvelle économie sociale, les Centres de la petite enfance valorisent d'abord le vote des usagers (75,3\% d'entre eux) puis celui des employés (59,6\% d'entre eux); la situation est différente dans la catégorie «Centres de travail adapté et aménagement forestier» et dans d'autres secteurs de la nouvelle économie sociale où plus souvent les employés votent (environ 23,5\% des entreprises) que les usagers (13,5\% d'entre elles, en moyenne) (Comeau et al., 2001: 69).

14 Dans le territoire de Chaudière-Appalaches, les 4101 personnes siégeant aux conseils d'administration se répartissent comme suit: membres individuels $(67,6 \%$ des organismes leur donnent un droit de vote), les usagers $(32,8 \%$ des organismes), les bénévoles (28,0\%), un ou des représentants d'organismes membres $(20,8 \%)$, le personnel $(18,4 \%)$, un ou des représentants d'organisations gouvernementales (16,0\%), un ou des représentants du milieu des affaires $(9,6 \%)$ et un ou des représentants du milieu syndical (1,6\%) (Comeau, 2003: 63).

15 En 2000, seulement 7\% des salariés du milieu communautaire québécois gagnaient plus de $22400 €$ par année (Bourdon et al., 2001: 26-27). Plus précisément, le salaire horaire moyen est de 8,28 € l'heure (Comeau, 2003: 74). En ce qui concerne les avantages sociaux, près de la moitié des organismes affirment offrir aux salariés des avantages qui dépassent les normes minimales du travail. Les deux principaux avantages offerts sont une assurance-groupe payée en partie par l'employeur (29,0\% des organismes de Chicoutimi et 33,2\% des organismes de Chaudière-Appalaches) et les congés sans solde (28,8\% des organismes de Chaudière-Appalaches); très peu d'organismes rendent accessible à leur personnel un régime collectif de retraite (6,5\% dans Chicoutimi, 7,0\% à Montréal et 4,4\% dans Chaudière-Applaches) (Tremblay et Tremblay, 1998: 83; Mathieu et al., 2001: 48; Comeau, 2003: 78).

${ }^{16}$ McMullen et Schellenberg (2003) arrivent à cette conclusion à partir de l'Enquête sur le lieu de travail et les employés faite en 1999 par Statistique Canada. L'Enquête de type longitudinal porte sur 6320 établissements et 23500 employé(e). Ces auteurs recourt à la notion de troisième secteur ou third sector, qui comprend, dans leur cas, une gamme d'organismes un peu plus large que celle entendue dans cet article, puisqu'elle comprend notamment les musées.

17 À Montréal, les organismes offraient annuellement, en 1993, $18230 €$ pour la fonction de coordination, $15846 €$ pour l'intervention et $13581 €$ pour le soutien technique (Mathieu et al., 2001: 49). Dans Chaudière-Appalaches, le taux horaire le plus élevé est accordé à la coordination (10,37€ l'heure). En moyenne, une personne salariée ayant un diplôme universitaire peut y gagner 10,09€ l'heure, comparativement à 6,15€ l'heure pour un employé sans diplôme secondaire (Comeau, 2003: 74). Par ailleurs, les variations salariales portent également sur les secteurs: le taux horaire est de 9,70€ dans les Centres de la petite enfance, de 6,85€ dans les autres initiatives de la nouvelle économie sociale et de 8,14 € dans le communautaire (Comeau 2003: 75).

${ }^{18}$ C'est la conclusion à laquelle conduisent les analyses statistiques réalisées dans Bourdon et al., (2001: 26), Comeau et al. (2001: 105) et Comeau (2003: 138). Pour leur part, Tremblay et Tremblay (1998: 65) pensent qu'il existe une «tendance à retrouver les femmes dans les emplois moins rémunérés»; or, ils omettent d'appliquer un calcul statistique à leurs données qui confirmerait ou non cette tendance. Nous avons appliqué le test du khi carré à leurs données et celui-ci révèle qu'il n’y a pas de différence significative entre les distributions des hommes et des femmes sur le plan salarial.

19 À Montréal, 6,0\% des organismes ont une accréditation syndicale (Mathieu et al., 2001: 48). Seulement 7,4\% des organismes communautaires du Québec sont syndiqués en l'an 2000; dans le secteur des services de garde, le taux de syndicalisation est de 29,5\%; il est de 12,5\% en insertion professionnelle et formation de la main-d'œuvre; $7,9 \%$ dans le domaine de l'éducation; $2,8 \%$ en santé et services sociaux; etc. (Bourdon et al., 2001: 19). Si la syndicalisation est faible, on remarque tout de même un certain effort pour codifier les conditions de travail puisque celles-ci sont explicitées dans une politique écrite dans la majorité des organismes (61,9\% d'entre eux) (Comeau, 2003: 71). 
la production des biens et la livraison des services.

Sur le plan organisationnel, on remarque, premièrement, que les systèmes associatifs locaux réalisent de bien des manières leur mission de bien-être. Ainsi, le communautaire affirme plus souvent rejoindre des personnes vivant un «problème» (maladie, faim, analphabétisme, pauvreté, violence familiale, toxicomanie) ou des populations spécifiques (jeunes, personnes âgées, femmes, sans abri, personnes handicapées $)^{20}$. De fait, plusieurs personnes connaissent de multiples problèmes qui découlent de leur situation de pauvreté $^{21}$. Certains types de services rejoignent davantage ou exclusivement les hommes ${ }^{22}$, mais dans l'ensemble, les femmes représentent la majorité des usagers ${ }^{23}$.

Deuxièmement, les services produits par les systèmes associatifs locaux compensent l'impossibilité ou l'incapacité de l'État à offrir certains types de services sur un territoire donné. Mises à part les contraintes budgétaires qui peuvent être évoquées, ces limites de l'État tiennent, d'une part, à la nature même des activités qui exigent la proximité des organismes et une implication intensive des personnes qui y interviennent et, d'autre part, aux difficultés qu'éprouvent des personnes à avoir accès aux services publics (à cause d'un manque d'information, de leur éloignement géographique, du rapport conflictuel qui existe entre elles et ces services, de la crainte de subir des sanctions, de la pauvreté ou de la nature même de leur problème qui nourrit les préjugés).

Troisièmement, on remarque, sur le plan organisationnel, qu'il s'agit habituellement d'organismes ayant très peu d'employés. Le communautaire regroupe des organismes comptant moins d'employés que ceux de la nouvelle économie sociale ${ }^{24}$.

Quatrièmement, on retrouve dans les systèmes associatifs locaux de bien-être une main-d'œuvre très majoritairement féminine ${ }^{25}$. L'importance numérique des femmes varie toutefois lorsque l'on considère les secteurs d'activités ${ }^{26}$. De manière générale, dans un secteur féminin, les fonctions d'encadrement, de soutien administratif et de production sont occupées par les femmes et ce, dans la même proportion que leur présence dans le secteur; dans les secteurs masculins, elles occupent plus souvent la fonction de soutien administratif ${ }^{27}$.

Cinquièmement, plusieurs indices laissent croire qu'une bonne partie des organismes des systèmes associatifs locaux de bien-être favorisent l'inclusion et l'autonomie des employés dans l'organisation du travail ${ }^{28}$. Ces situations d'ouverture se manifestent par: 1) la polyvalence (enrichissement et rotation des tâches intégrant notamment des activités de gestion et de production); 2) la possibilité pour les travailleurs de modifier

\footnotetext{
${ }^{20}$ Plus souvent que dans le communautaire, les organismes de la nouvelle économie sociale affirment ne pas viser des personnes ayant des caractéristiques particulières (Comeau, 2003: 90).

${ }^{21}$ Mercier et Métivier (2003: 51) remarquent que les usagers de l'aide alimentaire éprouvent des problèmes de santé physique (difficulté de se procurer des médicaments trop dispendieux, alimentation déficiente, toxicomanie, logement mal chauffé), de santé mentale (dépression, détresse liée aux problèmes financiers et à l'isolement, intégration sociale difficile), d'isolement social (exclusion du travail, faible estime de soi, analphabétisme, rapports conflictuels avec les institutions) et de relations sociales (conflits familiaux, ruptures, violence, placement d'enfants).

${ }^{22}$ C'est du moins ce que constatent Mercier et Métivier (2003: 40) dans une soupe populaire et au Service d'entraide de Moisson-Estrie.

${ }^{23}$ Ainsi, les organismes de Chaudière-Appalaches rejoignent directement et indirectement une «clientèle» constituée respectivement de 60,9\% et de 61,6\% de femmes (Comeau, 2003: 88).

${ }^{24}$ Dans Chaudière-Appalaches, un peu plus de la moitié des organismes ont quatre employés ou moins (Comeau, 2003: 96). En moyenne, on compte 15,5 employés par organisme; dans le communautaire, cette moyenne s'établit à 10,8 employés alors que dans la nouvelle économie sociale, elle est de 33,3 personnes (Comeau, 2003: 121). Tremblay et Tremblay (1998: 59) constatent, pour leur part, qu'en moyenne, cinq employés oeuvrent dans les organismes communautaires de Chicoutimi.

${ }_{25}$ Parmi les 171 employés des organismes communautaires de Chicoutimi ayant indiqué leur sexe, 72,9\% sont des femmes (Tremblay et Tremblay, 1998: 60). Dans Chaudière-Appalaches, 85,2\% des 1049 employés rejoints par l'enquête sont des femmes (Comeau, 2003: 110). Dans le Bas-Saint-Laurent, 65,3\% de la main-d'œuvre de l'économie sociale est constituée de femmes (Saucier et Thivierge, 1999: 41).

${ }^{26}$ Ainsi, la main-d'œuvre est féminine à $94,0 \%$ dans les Centres de la petite enfance, à 89,0\% dans les entreprises d'économie sociale en aide à domicile, à $25 \%$ dans la catégorie «Centres de travail adapté et aménagement forestier» et à 64,0\% dans la catégorie «autres» (coopératives funéraires, entreprises d'insertion, ressourceries, entre autres) (Comeau et al., 2001: 77).

${ }^{27}$ Dans les catégories «Centres de travail adapté et aménagement forestier» et «autres» (secteurs à main-d'œuvre masculine), plus de $80 \%$ des postes de soutien administratif sont occupés par des femmes, alors que leur présence dans la production se situe autour de $35 \%$ dans ces mêmes secteurs (Comeau et al., 2001: 78).

${ }^{28}$ Dans des entrevues de groupe, Mathieu et al. (2001: 50) constatent que les équipes de travail peuvent exercer une influence réelle dans les organismes et ils qualifient de «participatif» le mode de gestion qui y prévaut. Les monographies d'organismes sont également intéressantes pour saisir ce phénomène complexe de la participation des employés à la production. Voir à cet effet une analyse dans les secteurs de la coopération du travail et des services financiers (Comeau et al., 2002) et en aide à domicile (Comeau et Aubry, 2003).
} 
l'organisation des services; 3) le travail en équipe; et 4) la formation. Par ailleurs, il arrive que se produisent des situations de restriction à l'implication des employés: 1) une différenciation marquée de catégories de travailleurs à cause des tâches (bureau et production, par exemple) et des lieux de travail qualitativement différents (travail en industrie et à domicile pour la même coopérative); 2) le taylorisme qui se manifeste par un travail répétitif, la mesure du temps et des mouvements, la division sociale du travail (séparation des domaines de la décision et de l'exécution) et la division technique du travail (séparation et distinction étanche des sphères d'activités); 3) l'isolement des travailleurs qui ne permet pas une socialisation de la production; 4) la sous-traitance.

Sixièmement, les outils de gestion sont apparemment peu sophistiqués dans les systèmes associatifs. La prévision des activités prend forme dans un plan annuel et rarement dans des plans de communication ou des plans à plus long terme ${ }^{29}$.

Septièmement, sur le plan des sources de revenus, les organismes de ces systèmes reçoivent principalement une subvention de l'État ${ }^{30}$. En outre, une des particularités des systèmes associatifs locaux de bien-être est la contribution de bénévoles ${ }^{31}$. Enfin, il existe une différence marquée entre le communautaire et la nouvelle économie sociale quant aux sources de revenus, la dernière réussissant à générer davantage de revenus autonomes $^{32}$.

Huitièmement, les systèmes associatifs locaux possèdent des marges de manœuvre financière très réduites $^{33}$. Le manque de prévisibilité des ressources impose de la flexibilité à la main-d'œuvre et les revenus autonomes réussissent tant bien que mal à assurer certains aspects de l'infrastructure des organismes comme par exemple l'encadrement $^{34}$.

\section{L'Influence des Phénomènes Structurels}

D'après la théorie de la structuration (Giddens, 1997), il existe des phénomènes structurels d'ordres politiques, économiques et organisationnels qui s'imposent, d'une certaine manière, pour façonner un système associatif local de bien-être. D'autre part, il existe des conduites stratégiques adoptées par divers acteurs qui façonnent également ces systèmes. Si les phénomènes structurels conditionnent les conduites des acteurs, ceux-ci peuvent à leur tour exercer une influence sur les premiers, parce que leurs actions peuvent modifier les normes, l'exercice des contraintes et les représentations.

Les recherches consultées permettent d'identifier un certain nombre de phénomènes expliquant la diversité des systèmes associatifs locaux de bien-être. Parmi les phénomènes structurels, nous retenons le territoire, l'âge des organismes, leur taille et la nature même des services offerts. En premier lieu, à la notion de territoire nous associons les caractéristiques même de la région et la défavorisation plus ou moins grande de ses localités qui influencent la structuration des systèmes associatifs. Ainsi, dans une région telle Chaudière-Appalaches comprenant une composante rurale importante, les systèmes associatifs se distinguent de ceux en milieu urbain en couvrant plusieurs municipalités ${ }^{35}$; en milieu

${ }^{29}$ Les organismes recourent à un plan annuel dans 80,8\% des cas; moins de $10 \%$ des organismes élaborent un plan de communication ou un plan triennal (Comeau, 2003: 92).

30 Une grande partie des revenus (budget annuel moyen de $160000 €$ ) provient de l'État $(54,5 \%$ du budget des organismes de Chicoutimi d'après Tremblay et Tremblay [1998: 131] et 63,4\% du budget des organismes de Chaudière-Appalaches) et les possibilités de revenus autonomes sont plutôt restreintes (31,8\% des revenus générés par la vente des services et 4,8\% de dons et de donations) (Comeau, 2003: 97).

31 À Chicoutimi, on dénombre 37,8 bénévoles par organisme (Tremblay et Tremblay, 1998: 34). Dans Chaudière-Appalaches, 85,2\% des organismes comptent (en 2001) 39,4 bénévoles en moyenne; ce sont des femmes dans 75,7\% des cas (Comeau, 2003: 116).

32 En 2001, dans la région de Chaudière-Appalaches, un organisme communautaire moyen obtenait $18455 €$ de revenus autonomies, alors qu'une entreprise moyenne de la nouvelle économie sociale en comptait 114385 € (Comeau, 2003: 100).

33 Â Chicoutimi, $72 \%$ des organismes avouent avoir des difficultés de financement; par ailleurs, les organismes sacrifient jusqu'à $20 \%$ des heures de fonctionnement à la recherche de financement (Tremblay et Tremblay, 1998: 169). Dans Chaudière-Appalaches, les organismes y consacrent, en moyenne, 427,2 heures annuellement (Comeau, 2003: 103).

34 À cet effet, la part du budget annuel occupée par des revenus récurrents (assurés pour une période de trois ans) est de $44,1 \%$ en moyenne. Les difficultés de financement amènent les organismes (83,9\% d'entre eux) à mettre temporairement à pied 2,3 employés, en moyenne, pendant 26,8 semaines. Par ailleurs, les revenus autonomes tentent de compenser pour les choix faits par l'État en matière d'encadrement, puisque celui-ci privilégie l'encadrement des personnes en insertion $(40,7 \%$ du temps défrayé par un appui gouvernemental, comparativement à 16,4\% du temps d'encadrement des bénévoles et à 27,9\% du temps d'encadrement des employés couverts par l'État) (Comeau, 2003: 120).

${ }^{35}$ Cette comparaison entre une région donnée et l'ensemble du Québec a été rendue possible grâce à l'étude réalisée par le Comité sectoriel de la main-d'œuvre de l'économie sociale et de l'action communautaire, entre le l mars 2000 et le 16 mai 2000 (Comité sectoriel de la main-d'œuvre, 2000). Les données de cette étude ont été recueillies auprès de 2315 organismes à l'aide d'un sondage téléphonique portant sur leur vie démocratique, leurs finances et leur main-d'œuvre. L'étude réalisée dans Chaudière-Appalaches a repris les mêmes catégories de couverture géographique et a ainsi pu en faire la comparaison (Comeau, 2003: 39). 
fortement urbanisé, le champ de l'immigration occupe les systèmes associatifs locaux de bien-être ${ }^{36}$ alors que ce champ est à peu près absent dans les systèmes associatifs des régions à caractère rural, du moins au Québec. Par ailleurs, à l'intérieur même d'une région, les zones défavorisées accueillent des organismes ayant moins d'employés qu'ailleurs, des budgets plus modestes ${ }^{37}$ et une moindre capacité de recueillir des dons. En termes de retombées, ces organismes contribuent moins à la création d'emplois et à la constitution de réseaux que les organismes appartenant à des zones plus favorisées ${ }^{38}$. Entre les régions périphériques, d'une part, et les régions plus fortement urbanisées du centre, d'autre part, il existe également des différences statistiques importantes qui font que les systèmes associatifs urbains sont nettement plus avantagés en termes de ressources financière et humaine $^{39}$. En ce sens, le territoire où se déploie un système associatif représente un phénomène global (il comprend les dimensions économique, politique, sociale et culturelle d'une société), complexe (il regroupe plusieurs communautés et conditionne plusieurs aspects de la vie quotidienne) et lourd sur le plan organisationnel. En effet, son influence s'exerce à long terme et de manière déterminante, puisqu'il rend accessibles ou non des ressources humaines et matérielles.

En second lieu, l'âge des organismes représente un phénomène structurel exprimant un contexte de création et suppose d'abord un type de mission. En effet, les organismes récents adoptent des missions et des activités bien différentes d'organismes plus anciens ${ }^{40}$. Par ailleurs, la période de fondation d'un organisme suppose un appui spécifique de l'État qui favorise davantage, par ses interventions, une vague d'organismes plutôt associés au communautaire ou plutôt associés à la nouvelle économie sociale ${ }^{41}$, des secteurs tels la santé ou l'aide à la famille plutôt que la défense des droits, par exemple (Mathieu et al., 2001: 53). En outre, l'âge élevé d'un organisme suppose un degré d'institutionnalisation ${ }^{42}$ plus poussé, c'est-à-dire un niveau relativement élevé de formalisation et de codification des habitudes, des règles, des routines et des normes. Au contraire, un organisme récent risque plus souvent de devoir mettre en place des procédures, de créer des outils de planification et de développer un réseau qui est au départ plutôt embryonnaire. En outre, un organisme plus ancien possède habituellement un enracinement dans le milieu et une capacité de régénération acquise au cours des divers événements plus ou moins difficiles qui ont ponctué son histoire ${ }^{43}$.

En troisième lieu, la taille d'un organisme symbolise sa croissance et s'exprime notamment par un nombre relativement élevé d'employés de même qu'un budget et un avoir des sociétaires assez importants. La croissance d'un organisme sur ces

${ }^{36}$ Il s'agit d'un champ couvert par 9,2\% des organismes étudiés à Montréal par Mathieu et al. (2001: 27).

${ }^{37}$ Bien que Saucier et Thivierge (1999: 37) n'aient pas utilisé de test statistique pour confirmer cette tendance, les budgets moyens par organisme varient entre $48293 €$ pour la MRC du Témiscouata à $467232 €$ pour la MRC Rimouski-Neigette.

${ }^{38}$ C'est du moins ce qui ressort des comparaison statistiques faites sur ces variables et selon les indices de défavorisation des Municipalités régionales de comté (MRC) de Chaudière-Appalaches (Comeau, 2003).

${ }^{39}$ Une comparaison faite entre 126 organismes financés par le Fonds de lutte contre pauvreté, dont 63 en Gapsésie et 63 dans les quartiers populaires de Montréal, laisse voir des différences considérables entre ces systèmes associatifs locaux de bien-être (Comeau et al., 2002).

${ }^{40}$ Les organismes qui se spécialisent dans le «développement économique, création d'emplois et d'entreprises», la «(ré-)insertion professionnelle et employabilité», le «développement culturel» et le «regroupement, soutien et représentation d'organismes» sont plus récents que ceux qui se consacrent aux activités telles la «sensibilisation, promotion et défense des droits», l' «hébergement, répit et garde», la «réadaptation physique et intellectuelle», etc. (Comeau, 2003: 57).

${ }^{41}$ Les organismes des générations les plus anciennes, d'une part, et les plus récentes, d'autre part, sont plutôt associés à la nouvelle économie sociale. Dans Chaudière-Appalaches, les initiatives d'économie sociale les plus anciennes concernent les coopératives d'habitation et les Centres de travail adapté, par exemple. Quant aux initiatives récentes, il a déjà été mentionné que le gouvernement du Québec a été particulièrement actif pour ce qui est du développement de l'économie sociale, avec les mesures prises lors du Sommet sur l'économie et l'emploi (1996) et la Politique de développement régional et local (1997). Le communautaire connaît une vague de créations importante entre 1990 à 1996, une période caractérisée par le début, en 1991, de la Réforme du réseau de la santé et des services sociaux et la régionalisation (Comeau, 2003: 54).

${ }^{42}$ L'institutionnalisation est entendue ici en tant que processus de diffusion et de codification dans différents lieux ou instances, des principes et des règles relatives aux propositions promues par différents groupes sociaux plus ou moins en conflit. L'étude du processus d'institutionnalisation permet de comprendre comment des idées, actions ou structures deviennent des institutions, c'est-à-dire des règles, de nouvelles manières de faire et d'agir, dorénavant «tenues pour acquises» (taken for granted) et d'une «seconde nature» (second nature) (Bordt, 1997).

${ }^{43}$ Les analyses statistiques montrent que les organismes anciens ont plus souvent que les organismes récents une assemblée générale annuelle, une participation élevée des membres à celle-ci, une place pour les employés et les usagers dans leurs instances, des bénévoles plus nombreux et une plus grande capacité de générer des revenus provenant de la vente (sur ce dernier point, c'est le cas également de la nouvelle économie sociale «dernière vague»). Les organismes les plus anciens ont également constitué des réseaux comptant le plus d'interlocuteurs (Comeau, 2003: xvii). 
aspects signifie qu'il a connu un certain processus d'institutionnalisation. Les analyses statistiques ${ }^{44}$ démontrent que la taille relativement important d'un organisme est fortement associée à la création d'instances tel un lieu d'expression pour les usagers, sans doute à cause de la complexité qu'engendre la croissance d'un organisme. On remarque également que les plus gros organismes sont ceux qui rendent explicites les conditions de travail en les présentant dans une politique écrite. L'avancement d'un organisme en âge ne s'accompagne pas nécessairement d'une plus grande taille; il s'agit donc de deux phénomènes indépendants ${ }^{45}$.

En quatrième lieu, les liens d'usage des services produits touche la fréquentation des activités et la nature des services, et configure les organismes. Puisque le communautaire oriente davantage ses activités vers des problématiques sociales particulières, comme nous l'avons vu, la populations vivant ces problèmes est pauvre et n'a pas la capacité de payer pour les services. Ceci explique en partie pourquoi le communautaire tire le moins de revenus de la vente de services et qu'il embauche le moins de personnes ${ }^{46}$. Par ailleurs, la nature des services entraîne deux situations de fréquentation des usagers: les services auxquels recourent peu souvent un grand nombre de personnes (services d' «information, référence, documentation, recherche et conseil», entre autres) et ceux que fréquentent souvent un petit nombre de personnes (services de garde, notamment) ${ }^{47}$. On peut considérer, enfin, que la nature des services et le secteur d'activités spécifient une population particulière à rejoindre, un réseau d'acteurs potentiels et un univers sociotechnique limitant les choix, du moins à court terme, en matière de livraison des services et de leur usage.

\section{La Dynamique des Rapports entre les Acteurs: un phénomène stratégique et interactif central}

Les capacités stratégiques des organismes et la dynamique des rapports sociaux permettent d'expliquer un autre aspect de la structuration du système associatif local. La dynamique des rapports entre les acteurs et l'action des mouvements sociaux a été clairement constatée dans les analyses statistiques. En effet, la capacité qu'a un groupe de faire valoir son point de vue et ses intérêts dans la société et dans une organisation, peut amener des changements en sa faveur dans les ententes et les règles, et lui permettre d'acquérir de nouvelles ressources (Touraine, 1993). Les résultats ont montré que ces rapports concernent les organismes et l'État, la direction des organismes et le personnel, les hommes et les femmes, les organismes et d'autres acteurs locaux. Cette dynamique s'exerce à l'intérieur des organismes et vers l'extérieur. En ce sens, les organismes communautaires et de la nouvelle économie sociale sont à la fois l'expression des mouvements sociaux et sont soumis à leur pression. Vus sous l'angle de l'expression des mouvements sociaux, les systèmes associatifs locaux agissent comme un ensemble de mécanismes sociaux poussés par la société civile et empruntant des formes contractuelles pour résoudre les décalages qui existent entre les aspirations et les besoins non comblés, d'une part, et les règles institutionnalisées prévalant dans la société, d'autre part. Vus sous l'angle d'une cible des mouvements sociaux, les systèmes associatifs locaux intègrent peu à peu les propositions des mouvements sociaux à l'instar des entreprises privées et des entreprises publiques. En ce sens, les mouvements sociaux contribuent en partie à la construction (et à la

\footnotetext{
44 Lire à cet effet Comeau (2003)

45 La corrélation entre les variables de l'âge et de la taille n'est pas significative (Comeau, 2003: 49). On comprend que des processus de downsizing et de croissance accélérée peuvent se produire à tout moment dans l'histoire d'un organisme.

46 Effectivement, le communautaire compte, en moyenne, 10,8 employés alors que dans la nouvelle économie sociale, cette moyenne est de 33,3 (Comeau, 2003: 109).

47 Cette idée que des services rejoignent un grand nombre de personnes (référence et information, loisirs, etc.) et que d'autres accueillent moins de personnes qui reviennent fréquemment (hébergement, garde de la petite enfance, etc.) est formulée par Mathieu et al. (2001: 37) et Comeau (2003: 128-129).
} 
déconstruction) des conditions de sa propre existence (Bajoit, 2003: 141).

La dynamique des rapports sociaux explique pourquoi les employés ayant exercé des pressions (dans les Centres de la petite enfance, en l'occurrence) et ceux faisant valoir le mieux leurs compétences (les employés à la coordination et les plus scolarisés) bénéficient des meilleures conditions de travail ${ }^{48}$. On comprend également que la situation des femmes dans la société et leur expression dans le mouvement féministe se reflète dans les systèmes associatifs locaux de bien-être. Il s'agit d'un secteur de services où on trouve une majorité de femmes en tant qu'employées et en tant qu'administratrices. Proportionnellement, les femmes sont sous-représentées à la coordination de certains organismes, en particulier dans la nouvelle économie sociale où il existe des secteurs davantage masculins (par exemple, dans les centres de travail adapté). Par ailleurs, la structure des salaires est égalitaire entre les hommes et les femmes dans les organismes; à cet égard, l'équité promue par les mouvements sociaux se concrétise sur cet aspect. On peut croire que les femmes et les jeunes font entendre leurs besoins d'insertion dans la mesure où les postes d'insertion leur sont généralement attribués dans les organismes.

Les rapports entre les organismes et les établissements du milieu sont marqués par la collaboration et occasionnellement par la concurrence voire le conflit. Ainsi, le taux de roulement du personnel dans les organismes résulte de la compétition sur le marché de l'emploi que lui livrent les établissements publics tout particulièrement. Ceci peut expliquer pourquoi les organismes de Chaudière-Appalaches se démarque de leurs homologues du Québec ${ }^{49}$ avec, en proportion, pratiquement le double d'employés ayant moins de deux ans d'ancienneté, comme si le fait d'être situé près de la capitale nationale offrait des possibilités d'emplois dans la fonction publique et faisait que le système associatif local constituait une sorte de tremplin vers ce type d'emplois. Enfin, sur le plan stratégique, les organismes privilégient des rapports avec certains établissements. Le cas des Centres de la petite enfance est particulièrement révélateur. Le fait que ceux-ci entretiennent des contacts fréquents avec les ministères s'explique par l'appui financier important que l'État accorde aux Centres de la petite enfance et par les contraintes qu'il exerce sur eux, par ailleurs, à suivre une réglementation détaillée touchant les services de garde à l'enfance.

\section{Conclusion}

Cet article montre que lorsque l'on envisage les associations en tant que systèmes locaux de bien-être, ceux-ci prennent des configurations différentes. Sur le plan institutionnel, des systèmes démontrent davantage d'ouverture politique que d'autres; sur le plan organisationnel, des systèmes réunissent des ressources plus importantes et de meilleure qualité que d'autres. Sur ces plans, des phénomènes structurels (le territoire, l'âge des organismes, leur taille et la nature de leurs activités) ainsi qu'un phénomène stratégique central qu'est la capacité d'agir, structurent les systèmes associatifs locaux de bien-être.

Le caractère empirique de cette contribution permet de faire état de la situation contemporaine des systèmes associatifs locaux de bien-être. Il amène aussi à mettre en perspective certains jugements parfois rapides sur différents aspects de ces systèmes. L'analyse confirme également la présence de phénomènes structurels qui agissent à l'insu des acteurs mais ayant une influence sur la configuration de ces systèmes. Ces informations permettent enfin de soulever l'enjeu de la capacité d'innovation des systèmes associatifs locaux de bien-être face aux nombreux défis qu'ils rencontrent: l'autonomie et la reddition de comptes dans le cadre du financement étatique, le risque de substitution des services, la rétribution des ressources humaines, la mobilisation de la population et le changement social, entre autres. Les systèmes associatifs locaux de bien-être campent d'ailleurs leur légitimité sur cette capacité d'innovation sociale et justifient leur complémentarité au système public tout particulièrement, en

\footnotetext{
${ }^{48}$ Les tests statistiques concluants sont établis dans Comeau (2003: 74-75).

49 Encore une fois, les données relatives aux organismes du Québec proviennent d'une étude réalisée par le Comité sectoriel de la main-d'œuvre de l'économie sociale et de l'action communautaire (Comité sectoriel de la main-d'œuvre, 2000). L'étude réalisée dans Chaudière-Appalaches a repris les mêmes catégories et a ainsi pu en faire la comparaison (Comeau, 2003: 41).
} 
mettent en valeur leur apport unique pour les populations marginalisées.

La notion d'innovation sociale met en évidence l'inédit dans les manières de résoudre les questions sociales et les problèmes sociaux, et amène à considérer que ces nouvelles manières de faire conduisent à de nouvelles structures sociales, à nouveaux rapports sociaux, à de nouveaux modes de décisions, etc. (Chambon, David et Devevey, 1982: 8, 15). Au moins deux conditions prévalent pour que les systèmes associatifs locaux de bien-être puissent poursuivre l'innovation sociale: le risque et les réseaux. L'innovation constitue une situation à risque car, en premier lieu, l'élaboration et l'issue de l'innovation sociale sont incertaines (Thuderoz, 1997). En second lieu, en tant que démarche instituante, l'innovation sociale défie l'institué et menace de détruire l'ordre établi antérieurement (Schumpeter dans Alter, 1998). En ce sens, elle défait la tradition, dépasse la routine et défie les contraintes. En troisième lieu, au cours de son développement, l'incertitude plane à cause des nombreux choix qui s'offrent et des sanctions possibles associées aux diverses actions possibles
(Drais, 1998). L'innovation devient d'autant plus insécurisante qu'elle suscite de la résistance en entraînant des bouleversements en profondeur et en tentant de dépasser les «marges de tolérance» (Alter, 1998).

En plus de pouvoir assumer des risques pour innover, les systèmes associatifs locaux de bien-être doivent être connectés à des réseaux puisque l'innovation sociale est la manifestation d'un sujet en réseaux. Par définition, un réseau se donne peu de contraintes (Callon et Latour, cités par Alter, 1998; Thuderoz, 1997), possède un caractère informel et échappe au contrôle, car l'innovation suppose une certaine forme de transgression des règles et une prise de risque (Callon, 1999).

Risques et réseaux peuvent être envisagés dans le cadre de modalités de gestion qui permettent les adaptations, les transformations et les négociations des innovations sociales. C'est l'expérimentation qui permet de vérifier à petite échelle la mise en œuvre d'une idée sans prétention de diffuser cette idée, du moins pendant son cours.

\section{Bibliografia}

ALTER, N.,1998, «Quelques principes de l'analyse sociologique de l'innovation», Éducation permanente, no 134, pp. 21-34.

BAJOIT, G., 2003, Le changement social. Approches sociologiques des sociétés occidentales contemporaines, Paris, Armand Colin, 188 p.

BENFORD, R. et HUNT, S., 2001, «Cadrages en conflit», in CEFAÏ, D. et TROM, D., 2001 (dir.), Les formes de l'action collective, Paris, Éditions de l'École des Hautes Études en Sciences sociales, pp. 163-187.

BORDT, R. L. 1997, "How Alternative Ideas Become Institutions: The Case of Feminist Collectives", Nonprofit and Voluntary Sector Quarterly, vol. 26, no 2, pp. 132-155.

BOURDON, S., DESCHENAUX F., COALLIER, J-C, DIONNE, P. et LECLERC, M-H, 2001, Le travail et les conditions de travail dans les organismes communautaires québécois. Rapport de l'enquête 2000, Sherbrooke, Université de Sherbrooke/ Collectif de recherche sur les occupations, $29 \mathrm{p}$.

CALON, M., 1999, «Entretien avec Michel Callon réalisé par Robert Lhomme et Jean Fleury», Recherche et Formation, n. ${ }^{\circ}$ 31 , pp. 113-126.
CHAMBON, J-L, DAVID, A. et DEVEVEY, J-M, 1982, Les innovations sociales, Paris, Presses Universitaires de France, Collection Que Sais-Je?, 128 p.

COMEAU, Y., 2003, Le communautaire, la nouvelle économie sociale et leurs retombées en région. Chaudière-Appalaches, L'Islet, Éditions Terres Fauves, 199 p.

COMEAU, Y. et AUBRY, F., 2003, «Les rapports de travail et la participation des employées» in VAILLANCOURT, Y. JETTÉ, C. et AUBRY, F. (dir.), L'économie sociale dans les services à domicile, Québec, Presses de l'Université du Québec, pp. 201-233.

COMEAU, Y., BEAUDOIN A., CHARTRAND-BEAUREGARD, J. HARVEY, M-E, MALTAIS, D. SAINT-HILAIRE, C. SIMARD, P. et TURCOTTE, D., 2001, L'économie sociale et le Plan d'action du Sommet sur l'économie et l'emploi, Québec, Centre de recherche sur les services communautaires, Université Laval et ÉNAP, 277 p.

COMEAU, Y., BOUCHER, J., MALO, M-C et VAILLANCOURT, Y., 2002, «Las configuraciones de las iniciativas de la economía social y solidaria», Cayapa (Vénézuela), vol. 2, no 3 , pp. 14-36.

COMEAU, Y., TURCOTTE, D., BEAUDOIN, A., VILLENEUVE J-P, J. BOUCHARD, M. LEVESQUE, B., RONDOT, S., MENDELL, M., 2002, Les effets du financement étatique sur 
les organismes communautaires: le cas du Fonds de lutte contre la pauvreté, Québec, Les Éditions Sylvain Harvey, 225 pages.

COMITÉ SECTORIEL DE LA MAIN-D'EUVRE, 2000, «Les premiers résultats de la plus vaste étude statistique jamais réalisée sur l'action communautaire et l'économie sociale», Recto Verso, no 286, pp. 13-16.

DRAIS, E.,1998, «Au risque d'innover. Une tripe articulation entre risque et innovation», Éducation permanente, no 134 , pp. 55-65.

ESPING-ANDERSEN, G., 1999, Les trois mondes de l'État-Providence, Paris, Presses Universitaires de France, 310 p.

ESPING-ANDERSEN, G., 1996, "After the Golden Age? Welfare State Dilemnas in a Global Economy", in ESPING-ANDERSEN, G. (dir.), Welfare States in Transition, Thousand Oaks, Sage, pp. 1-31.

GIDDENS, A.,1997, The Constitution of Society. Outline of the Theory of Structuration, Cambridge, Polity Press, 402 p.

LAVILLE, J-L et SAINSAULIEU, R.(dir.), 1997, Sociologie de l'association, Paris, Desclée de Brouwer, 403 p.

MATHIEU, R., VAN SCHENDEL, V. TREMBLAY, D. G. JETTÉ, C., DUMAIS, L. et CRÉMIEUX, P-Y, 2001, L'impact socio-économique des organismes communautaires et du secteur de l'économie sociale dans quatre arrondissements de la Ville de Montréal, Montréal, Université du Québec à Montréal/ /Laboratoire de recherche sur les pratiques sociales/TÉLUQ, $144 \mathrm{p}$.

MAYER, R., 2002, Évolution des pratiques en service social, Boucherville, Gaëtan Morin Éditeur, 489 p.

MERCIER, Clément et Annick MÉTIVIER (2003), Action intersectorielle et développement local à Sherbrooke: impact et perspectives pour l'action communautaire, Sherbrooke, Moisson-Estrie, 146 p.

MCMULLEN, K. et SCHELLENBERG, G., 2003, Job Quality In Non-Profit Organizations, Ottawa, Canadian Policy Research Networks (CPRN), CPRN Research Series on Human Resources in the Non-profit Sector, no 2, 75 p.

SAUCIER, C. et THIVIERGE, N., 1999, Un portrait de l'économie sociale au Bas-Saint-Laurent, Rimouski, Conseil régional de concertation et de développement/Comité régional d'économie sociale/Université du Québec à Rimouski, $154 \mathrm{p}$.

THUDEROZ, C., 1997, «L'usine et le petit pot. Pour une sociologie de l'innovation industrielle», Sociologie du travail, no 3, pp. 347-369.

TOURAINE, A., 1993, Production de la société, Paris, Seuil, $477 \mathrm{p}$.

TREMBLAY, M. et TREMBLAY, P-A, 1998, Les contributions sociales et économiques des organismes communautaires de la Corporation de développement communautaire du ROC, Chicoutimi, Université du Québec à Chicoutimi/Groupe de recherche en intervention régionale, $218 \mathrm{p}$.

VAILLANCOURT, Y., 2002, «Le modèle québécois de politiques sociales et ses interfaces avec l'union sociale canadienne», Enjeux Publics, vol. 3, no 2, 52 p.

VIVERET, P., 2001, Rapport d'étape de la mission «Nouveaux facteurs de richesse», Paris, Secrétariat d'État à l'économie sociale, $44 \mathrm{p}$.

WHITE, D.,1994, La gestion communautaire de l'exclusion, Lien Social et Politiques-RIAC, 32, Automne 1994, pp. 37-51. 